\title{
Efeitos genéticos aditivos e não aditivos, grupo genético e sistema de acasalamento nas características métricas da carcaça de novilhos oriundos do cruzamento alternado contínuo Charolês x Nelore
}

\author{
Additive and non-additive genetic effects, genetic group and \\ breeding system on carcass metric characteristics of steers \\ originated from Charolais $x$ Nellore rotational crossbreeding
}

\author{
Paulo Santana Pacheco ${ }^{1 *}$; João Restle ${ }^{2}$; Fabiano Nunes Vaz ${ }^{2}$; Ivan Luiz Brondani1; \\ Dari Celestino Alves Filho'; Magali Floriano da Silveira ${ }^{3}$; \\ Luciane Rumpel Segabinazzi ${ }^{4}$; Leandro da Silva Freitas ${ }^{5}$; \\ Marcelo Machado Severo ${ }^{6}$; André Fograça Nigeliskii ${ }^{6}$
}

\section{Resumo}

Foram avaliados os comprimentos de carcaça, de perna e de braço das carcaças de 876 novilhos oriundos de gerações sucessivas do cruzamento rotativo entre as raças Charolesa $(\mathrm{C})$ e Nelore $(\mathrm{N})$. Na análise dos dados consideraram-se dois modelos. No Modelo I, os efeitos genéticos foram representados por sistema de acasalamento - SA e grupo genético dentro de sistema de acasalamento - GG(SA), sendo: $\mathrm{C}$ e $\mathrm{N}$ (definidos), $1 / 2 \mathrm{C} 1 / 2 \mathrm{~N}$ e $1 / 2 \mathrm{~N} 1 / 2 \mathrm{C}(\mathrm{G} 1), 3 / 4 \mathrm{C} 1 / 4 \mathrm{~N}$ e $3 / 4 \mathrm{~N} 1 / 4 \mathrm{C}(\mathrm{G} 2), 5 / 8 \mathrm{C} 3 / 8 \mathrm{~N}$ e $5 / 8 \mathrm{~N} 3 / 8 \mathrm{C}(\mathrm{G} 3)$ e 11/16C5/16N e 11/16N5/16C(G4). O Modelo II correspondeu ao Modelo I, no entanto, substituindo $\mathrm{SA}$ e $\mathrm{GG}(\mathrm{SA})$ pelas covariáveis representando a percentagem da raça $\mathrm{C}$ no novilho (individual) e na sua mãe (materno) (efeito genético aditivo da raça $\mathrm{C}$ em relação à $\mathrm{N}$ ), e a percentagem de heterozigose no novilho e na sua mãe (efeito genético não-aditivo devido à dominância). Das características métricas da carcaça avaliadas, o comprimento de carcaça foi o mais influenciado pelos efeitos genéticos aditivos raciais e heteróticos, sendo o componente individual de maior importância do que o materno. A raça Charolesa apresentou maior comprimento de carcaça e menores de perna e de braço do que a raça Nelore. A heterose foi positiva e, quando expressa em percentagem, os valores foram muito próximos para todas as características métricas. Os possíveis efeitos genéticos não aditivos representados por epistasia e ligação gênica influenciaram as características da carne avaliadas. Os modelos avaliados se apresentaram similares quanto à eficiência de predição das características. $\mathrm{O}$ sistema de cruzamento utilizado é beneficiado pelos efeitos de heterose e complementaridade racial nas características métricas da carcaça de novilhos submetidos a sistema de produção visando o abate aos dois anos de idade.

Palavras-chave: Bos indicus, Bos taurus, comparação de modelos, produção de novilho jovem, regressão múltipla, sistemas de cruzamento

\footnotetext{
${ }^{1}$ Profs., Universidade Federal de Santa Maria, UFSM, Santa Maria, RS. E-mail: pacheco.dz.ufsm@hotmail.com; ivanbrondani@ gmail.com; darialvesfilho@hotmail.com

2 Pesquisadores, Universidade Federal de Tocantins, UFT, Araguaína, TO. E-mail: jorestle@terra.com.br; fabianonunesvaz@ gmail.com

3 Prof ${ }^{a}$, Universidade Tecnológica Federal do Paraná, UTFPR, Dois Vizinhos, PR. E-mail: mg_flor@yahoo.com.br

${ }^{4}$ Prof ${ }^{a}$, Universidade Federal do Pampa, UNIPAMPA, Dom Pedrito, RS. E-mail: lucianesegabinazzi@unipampa.edu.br

5 Prof., Instituto Federal Farroupilha, IFF, Alegrete, RS. E-mail: freitaszoot@hotmail.com

${ }^{6}$ Discentes, UFSM, Santa Maria, RS. E-mail: marcelozoot9@yahoo.com.br; fogaca.zootecnia@hotmail.com

* Autor para correspondência
} 


\begin{abstract}
Carcass, leg and arm lengths of 876 steers originated from rotational crossbreeding between Charolais (C) and Nellore $(\mathrm{N})$ breeds were evaluated. Two models were used in the analyses. In Model I, genetic effects were represented by breeding system - SA and genetic group nested within SA - GG(SA), being: $\mathrm{C}$ and $\mathrm{N}$ (straightbreds), $1 / 2 \mathrm{C} 1 / 2 \mathrm{~N}$ and $1 / 2 \mathrm{~N} 1 / 2 \mathrm{C}(\mathrm{G} 1), 3 / 4 \mathrm{C} 1 / 4 \mathrm{~N}$ and $3 / 4 \mathrm{~N} 1 / 4 \mathrm{C}(\mathrm{G} 2), 5 / 8 \mathrm{C} 3 / 8 \mathrm{~N}$ and $5 / 8 \mathrm{~N} 3 / 8 \mathrm{C}(\mathrm{G} 3)$ and $11 / 16 \mathrm{C} 5 / 16 \mathrm{~N}$ and $11 / 16 \mathrm{~N} 5 / 16 \mathrm{C}(\mathrm{G} 4)$. Model II corresponded to Model I, however, $\mathrm{SA}$ and $\mathrm{GG}(\mathrm{SA})$ were substituted by the covariables corresponding to the percentage of $\mathrm{C}$ breed of the steer (individual) and its mother (maternal) (additive genetic effect of $\mathrm{C}$ breed in relation to $\mathrm{N}$ ), and the percentage of heterozygosis of the individual and its mother (non-additive genetic effect due to dominance). From the evaluated metric carcasses characteristics, carcass length was the most influenced by additive due to breed and heterotic genetic effects, being the individual component of more importance than maternal. $\mathrm{C}$ steers showed higher carcass length and lower leg and arm lengths than N. Heterosis was positive and when expressed in percentage, the values were similar for all metric carcasses characteristics. The possible non-additive genetic effects, represented by epistasis and linkage influenced the characteristics. The models were similar with respect to capacity of prediction of characteristics. The crossbreeding system used took advantage of heterosis and breed complementarity, on the carcass metric characteristics of steers submitted to production system with purpose of slaughter at two years of age.
\end{abstract}

Key words: Bos indicus, Bos taurus, crossbreeding systems, model comparisons, multiple regression, production of young steer

\section{Introdução}

Em estudos de cruzamento de bovinos de corte, se considerado o sistema como um todo, não apenas a avaliação das características reprodutivas, de desempenho pré e pós-desmame são importantes, mas também o monitoramento do produto final, representadas pelas características da carcaça e carne. Isto porque a diversidade de raças e sistemas de cruzamento existentes pode resultar em variações nas características da carcaça e da carne (GREGORY; CUNDIFF, 1980; KOGER, 1980; DeROUEN et al., 1992), e isto deve ser considerado quando da adoção do cruzamento, visando atender necessidades específicas ou conjuntas de produtores, indústrias e consumidores (PEROTTO; ABRAHÃO; KROETZ, 2001; PEROTTO; MOLETTA; LESSKIU, 2002).

Das características quantitativas da carcaça avaliadas em estudos conduzidos com bovinos de corte, têm-se as métricas, que apresentam correlação positiva com peso de carcaça (MENEZES et al., 2005) e, consequentemente, com peso de abate, sendo estas duas últimas de grande importância comercial.
Estudos conduzidos no Brasil, envolvendo o cruzamento entre Charolês e Nelore (RESTLE et al., 2000; VAZ; RESTLE; ALVES FILHO, 2002; MENEZES et al., 2005), demonstraram que existem diferenças significativas no comprimento de carcaça, de perna e de braço de carcaças de novilhos de grupos genéticos definidos e cruzados comparados dentro de sistema de acasalamento, assim como entre sistemas de acasalamento, resultando em heterose positiva.

Quanto às estimativas dos efeitos genéticos aditivos raciais e não aditivos heteróticos, poucos são os estudos que avaliaram características métricas da carcaça, como o de Neville Junior, Mullinix Junior e McCormick (1984), indicando influência destes efeitos genéticos no comprimento de carcaça.

Objetivou-se com este estudo, avaliar os efeitos genéticos aditivos devido à raça e não aditivos heteróticos, grupo genético e sistema de acasalamento nos comprimentos de carcaça, de perna e de braço de carcaças de novilhos oriundos de gerações sucessivas do cruzamento rotativo Charolês x Nelore, abatidos aos dois anos de idade. 


\section{Material e Métodos}

O experimento de campo foi conduzido no Departamento de Zootecnia da Universidade Federal de Santa Maria - RS. Foi avaliada a carcaça de 876 novilhos nascidos entre 1986 e 2001, castrados aos sete meses de idade, oriundos de gerações sucessivas do cruzamento alternado (rotativo) contínuo entre as raças Charolesa e Nelore. Os novilhos eram filhos de vacas com idade variando entre 3 e 15 anos. Os animais foram submetidos a manejo nutricional visando o abate aos dois anos de idade, consistindo de recria em pastagem de verão e inverno, e terminação em confinamento.

Previamente à pesagem final de abate e o embarque para o matadouro, os animais foram submetidos a jejum de sólidos por período de 14 horas. Em seguida, foram transportados e abatidos seguindo o fluxo normal da indústria frigorífica.

Após o abate, as duas meias-carcaças foram identificadas e encaminhadas à câmara de resfriamento. Após o período de resfriamento por 24 horas sob temperatura oscilando entre zero e $2{ }^{\circ} \mathrm{C}$, as carcaças foram pesadas para obtenção do peso de carcaça fria.

$\mathrm{Na}$ meia carcaça fria direita, foram avaliadas as características métricas, sendo: comprimento de carcaça, tomada do bordo cranial medial da primeira costela ao bordo anterior do osso púbis; comprimento de perna, correspondente à distância entre o bordo anterior do osso púbis e a articulação tíbio-tarsiana; e comprimento de braço, medido da articulação rádio carpiana até a extremidade do olécrano.

A análise estatística foi realizada utilizando-se o procedimento GLM do software estatístico SASStatistical Analysis System (2013).

Os dados foram previamente submetidos ao teste de normalidade, através da estatística $W$ de Shapiro e Wilk (1965).

Foram considerados dois modelos denominados Modelo I e Modelo II. No Modelo I, os efeitos genéticos aditivos devido à raça e não aditivos (dominância, sobredominância, epistasia e ligação gênica) foram representados pelos efeitos de sistema de acasalamento do novilho - SA e grupo genético do novilho aninhado em sistema de acasalamento GG(SA).

O Modelo I foi assim descrito:

$$
\begin{aligned}
& \mathrm{Y}_{\mathrm{i} \ldots \mathrm{n}}=\mu+\beta_{1} \mathrm{IA}_{\mathrm{i}}+\beta_{2} \mathrm{IV}_{\mathrm{j}}+\beta_{3} \mathrm{IV}_{\mathrm{j}}+\mathrm{ANO}_{\mathrm{k}}+[ \\
& \left.\mathrm{SA}_{1}+\mathrm{GG}_{\mathrm{m}}(\mathrm{SA})_{1}\right]+\varepsilon_{\mathrm{i} . . \mathrm{n}}
\end{aligned}
$$

em que:

$\mathrm{Y}_{\mathrm{i} \ldots \mathrm{n}}=$ variáveis dependentes; $\mu=$ média geral; $\mathrm{IA}_{\mathrm{i}}=$ covariável idade de abate do novilho (em dias); $I V_{j}, I V 2_{j}=$ covariáveis idade da mãe do novilho ao parto (em anos) linear e quadrático, respectivamente; $\beta_{1}=$ coeficiente de regressão linear na idade de abate do novilho; $\beta_{2}$ e $\beta_{3}=$ coeficientes de regressão linear na idade da mãe do novilho ao parto linear e quadrático, respectivamente; $\mathrm{ANO}_{\mathrm{k}}$ $=$ ano de nascimento do novilho; $\mathrm{SA}_{1}=$ sistema de acasalamento do novilho; $\mathrm{GG}_{\mathrm{m}}(\mathrm{SA})_{\mathrm{l}}=$ grupo genético do novilho aninhado em sistema de acasalamento; $\varepsilon_{\mathrm{i} . . \mathrm{n}}=$ erro aleatório associado a cada observação, $\operatorname{NID}\left(0, \sigma^{2}\right)$.

Para a característica comprimento de braço, a covariável idade da mãe do novilho ao parto quadrático foi removida do modelo, pois $\mathrm{P}(\mathrm{F})>$ 0,05 .

Foi realizada análise de variância sendo aplicados o teste F para avaliar os efeitos do modelo e o teste $|\mathrm{t}|$ as estimativas dos coeficientes linear e angular. No Modelo II, os efeitos genéticos SA e GG(SA) do Modelo I foram substituídos pelos efeitos aditivos devido à raça e não aditivos devido à dominância, separados nos componentes individual e materno. Para isto, de uso de regressão múltipla, utilizaram-se covariáveis representadas pelas proporções da raça Charolesa (efeito genético aditivo devido à raça) e pelas heterozigoses (efeito genético heterótico devido à dominância) no novilho (individual) e na sua mãe (materno), conforme apresentado na Tabela 1. 
Tabela 1. Covariáveis utilizadas na estimação dos efeitos genéticos no Modelo II (genético aditivodominante).

\begin{tabular}{|c|c|c|c|c|c|}
\hline \multirow{2}{*}{$\begin{array}{c}\text { Sistema de } \\
\text { acasalamento }\end{array}$} & \multirow{2}{*}{ Grupo genético do novilho } & \multicolumn{4}{|c|}{ Covariáveis dos efeitos genéticos * } \\
\hline & & $\mathrm{Ci}$ & $\mathrm{Cm}$ & $\mathrm{HZi}$ & $\mathrm{HZm}$ \\
\hline \multirow[t]{2}{*}{ Definidos } & Charolês (C) & 1,000 & 1,000 & 0,000 & 0,000 \\
\hline & Nelore $(\mathrm{N})$ & 0,000 & 0,000 & 0,000 & 0,000 \\
\hline \multirow[t]{2}{*}{ G1 } & $1 / 2 \mathrm{C} 1 / 2 \mathrm{~N}$ & 0,500 & 0,000 & 1,000 & 0,000 \\
\hline & $1 / 2 \mathrm{~N} 1 / 2 \mathrm{C}$ & 0,500 & 1,000 & 1,000 & 0,000 \\
\hline \multirow[t]{2}{*}{$\mathrm{G} 2$} & $3 / 4 \mathrm{C} 1 / 4 \mathrm{~N}$ & 0,750 & 0,500 & 0,500 & 1,000 \\
\hline & $3 / 4 \mathrm{~N} 1 / 4 \mathrm{C}$ & 0,250 & 0,500 & 0,500 & 1,000 \\
\hline \multirow[t]{2}{*}{ G3 } & $5 / 8 \mathrm{C} 3 / 8 \mathrm{~N}$ & 0,625 & 0,250 & 0,750 & 0,500 \\
\hline & $5 / 8 \mathrm{~N} 3 / 8 \mathrm{C}$ & 0,375 & 0,750 & 0,750 & 0,500 \\
\hline \multirow[t]{2}{*}{ G4 } & $11 / 16 \mathrm{C} 5 / 16 \mathrm{~N}$ & 0,687 & 0,375 & 0,625 & 0,750 \\
\hline & $11 / 16 \mathrm{~N} 5 / 16 \mathrm{C}$ & 0,313 & 0,625 & 0,625 & 0,750 \\
\hline \multirow[t]{2}{*}{ G5 } & $21 / 32 \mathrm{C} 11 / 32 \mathrm{~N}$ & 0,656 & 0,313 & 0,687 & 0,625 \\
\hline & $21 / 32 \mathrm{~N} 11 / 32 \mathrm{C}$ & 0,344 & 0,687 & 0,687 & 0,625 \\
\hline \multirow[t]{2}{*}{ Rotativo em equilíbrio } & $2 / 3 \mathrm{C} 1 / 3 \mathrm{~N}$ & 0,667 & 0,333 & 0,667 & 0,667 \\
\hline & $2 / 3 \mathrm{~N} 1 / 3 \mathrm{C}$ & 0,333 & 0,667 & 0,667 & 0,667 \\
\hline
\end{tabular}

* $\mathrm{Ci}, \mathrm{Cm}$ = percentagem da raça Charolesa no indivíduo e na sua mãe, respectivamente; HZi, HZm = percentagem de heterozigose no indivíduo e na sua mãe, respectivamente.

Fonte: Elaboração dos autores.

Considerou-se o efeito genético aditivo como sendo o desvio da raça Charolesa em relação à raça Nelore.

O Modelo II foi assim descrito:

$$
\begin{aligned}
& \mathrm{Y}_{\mathrm{i} . \mathrm{n}}=\beta_{0}+* * *+\left[\beta_{4} \mathrm{Ci}_{4}+\beta_{5} \mathrm{Cm}_{5}+\beta_{6} \mathrm{HZi}_{6}+\right. \\
& \left.\beta_{7} \mathrm{HZm}_{7}\right]+\varepsilon_{\mathrm{i} . . \mathrm{n}} \\
& \text { em que: }
\end{aligned}
$$

$\mathrm{Y}_{\mathrm{i} \ldots \mathrm{n}}=$ variáveis dependentes; $\beta_{0}=$ intercepto; *** = conjunto de efeitos iguais aos do Modelo I; $\mathrm{Ci}_{4}, \mathrm{Cm}_{5}=$ covariáveis representadas pela percentagem da raça Charolesa no novilho e na sua mãe, respectivamente (Tabela 1); $\mathrm{HZi}_{6}, \mathrm{HZm}_{7}$ = covariáveis representadas pela percentagem de heterozigose no novilho e na sua mãe, respectivamente (Tabela 1); $\beta_{4}, \beta_{5}=$ coeficientes de regressão linear nas percentagens individual e materna da raça Charolesa, respectivamente; $\beta_{6}, \beta_{7}$ $=$ coeficientes de regressão linear nas heterozigoses individual e materna, respectivamente; $\varepsilon_{i \ldots \mathrm{n}}=$ erro aleatório associado a cada observação, NID (0, $\left.\sigma^{2}\right)$.
Estimativas das heteroses e contrastes, na mesma unidade de medida da característica, foram obtidas utilizando o procedimento GLM (SAS, 2013), para ambos os modelos.

A descrição do cálculo da heterose em percentagem, dos efeitos genéticos aditivos (individual e materno) devido à raça para o Modelo $\mathrm{I}$, e as heterozigoses individual e materna para o Modelo II constam em Pacheco et al. (2010).

Os valores preditos com o Modelo II para os sistemas de acasalamento e grupos genéticos avaliados, assim como para os não avaliados (G5 e Rotativo em equilíbrio), foram obtidas pela seguinte equação:

$$
\hat{y}=\mu N+\left[\beta_{4} C i_{4}+\beta_{5} C m_{5}+\beta_{6} H Z i_{6}+\beta_{7} H Z m_{7}\right]^{*}
$$

em que:

$\hat{y}=$ valor predito da característica; $\mu N=$ média ajustada da raça Nelore para a característica; * = conjunto de coeficientes descritos no Modelo II anteriormente. 
A descrição da análise do diagnóstico de multicolinearidade entre as variáveis preditoras (covariáveis) dos efeitos genéticos do Modelo II (Ci, Cm, HZi, HZm) consta em Pacheco et al. (2010).

A estimativa da proporção da contribuição conjunta dos efeitos genéticos não aditivos representados por epistasia e ligação gênica foi obtida através da redução na soma de quadrados do erro entre os modelos I e II, e analisada pelo teste F, conforme descrito por Dillard, Rodriguez e Robinson (1980). A descrição detalhada dos cálculos consta em Pacheco et al. (2010).

Além disso, para cada característica, comparouse a eficiência de predição dos Modelos I e II por meio dos valores dos coeficientes de determinação $\left(\mathrm{R}^{2}\right)$ e de correlação de Pearson (r), conforme sugestão de Arthur, Hearshaw e Stephenson (1999).

\section{Resultados e Discussão}

A análise demonstrou que a multicolinearidade não influenciou na estimação dos coeficientes de regressão dos efeitos genéticos incluídos no Modelo II. Os resultados que mostram as estimativas dos efeitos genéticos aditivos raciais (expresso como desvio da raça Charolesa em relação à Nelore) e não aditivos heteróticos (devido à dominância), separados nos componentes individual e materno, de acordo com a característica métrica da carcaça avaliada (Tabela 2) indicam concordância nas estimativas dos efeitos genéticos obtidas pelos Modelos I e II. Entretanto, menores erros-padrão das estimativas foram verificados para as do Modelo II.

Tabela 2. Estimativas ${ }^{1}$ e erros-padrão dos efeitos genéticos aditivos devido à raça e heteróticos devido à dominância, de acordo com o modelo (Modelo I: genotípico; Modelo II: genético aditivo-dominante).

\begin{tabular}{llcccc}
\hline Modelo & \multicolumn{1}{c}{ Característica } & $\begin{array}{c}\text { Aditivo } \\
\text { individual }\end{array}$ & Aditivo materno & $\begin{array}{c}\text { Heterótico } \\
\text { individual }\end{array}$ & $\begin{array}{c}\text { Heterótico } \\
\text { materno }\end{array}$ \\
\hline \multirow{2}{*}{ Modelo I } & C. carcaça, cm & $8,78 \pm 1,03^{*}$ & $0,03 \pm 0,87$ & $3,73 \pm 0,55^{*}$ & \\
& C. perna, cm & $-2,96 \pm 0,80^{*}$ & $-1,07 \pm 0,67$ & $2,22 \pm 0,43^{*}$ & \\
& C. braço, cm & $-1,36 \pm 0,44^{*}$ & $-0,53 \pm 0,37$ & $1,10 \pm 0,24^{*}$ & \\
Valor t & C. carcaça, cm & 8,5 & 0,04 & 6,7 & \\
calculado & C. perna, cm & 3,7 & 1,5 & 5,1 & \\
& C. braço, cm & 3,1 & 1,4 & 4,7 & \\
\multirow{2}{*}{ Modelo II } & C. carcaça, cm & $7,15 \pm 0,68^{*}$ & $1,44 \pm 0,62^{*}$ & $4,47 \pm 0,49^{*}$ & $2,32 \pm 0,45^{*}$ \\
& C. perna, cm & $-3,87 \pm 0,53^{*}$ & $-0,28 \pm 0,48$ & $2,80 \pm 0,38^{*}$ & $0,82 \pm 0,35^{*}$ \\
\multirow{2}{*}{ Valor t } & C. braço, cm & $-2,45 \pm 0,29^{*}$ & $0,37 \pm 0,26$ & $1,53 \pm 0,21^{*}$ & $0,39 \pm 0,19^{*}$ \\
calculado & C. carcaça, cm & 10,4 & 2,3 & 9,2 & 5,1 \\
& C. perna, cm & 7,3 & 0,6 & 7,4 & 2,3 \\
\hline
\end{tabular}

${ }^{1}$ Para o Modelo II os valores representam as estimativas $\left(\beta_{4}, \beta_{5}, \beta_{6}\right.$, e $\left.\beta_{7}\right)$ das covariáveis dos coeficientes genéticos $(\mathrm{Ci}, \mathrm{Cm}, \mathrm{HZi}$ e $\mathrm{HZm}$, respectivamente). $* \mathrm{P}<0,05 ; * * \mathrm{P}<0,10$ pelo teste $|\mathrm{t}|$.

Graus de liberdade do erro Modelo $\mathrm{I}=848$.

Graus de liberdade do erro Modelo II = 854.

Fonte: Elaboração dos autores. 
Com o Modelo II, as estimativas de todos os efeitos genéticos foram de sinal positivo para comprimento de carcaça. Isto indica que quanto maior a proporção da raça Charolesa no genótipo do novilho e no da mãe deste, assim como quanto maior a heterozigose do novilho e de sua mãe, espera-se carcaça com maior comprimento. No estudo de Neville Junior, Mullinix Junior e McCormick (1984), que estimaram os efeitos genéticos aditivos raciais expressos como desvio das raças Angus e Santa Gertrudis em relação à Polled Hereford e não aditivos, verificou-se efeito genético aditivo individual da raça Santa Gertrudis $(4,9 \mathrm{~cm})$ e heterótico materno $(3,0 \mathrm{~cm})$ positivos para comprimento de carcaça.

Analisado o desempenho em confinamento de animais puros das raças Tabapuã e mestiços $1 / 2$ Bonsmara $+1 / 2$ Nelore ou $1 / 2$ Bonsmara $+1 / 4$ Red Angus + 1/4 Nelore, Clímaco et al. (2011) observaram que os melhores ganho de peso e conversão alimentar dos mestiços em relação aos zebuínos foi resultado da heterose individual nos $1 / 2$ Bonsmara $1 / 2$ Nelore e individual e materna nos $1 / 2$ Bonsmara $1 / 4$ Red Angus $1 / 4$ Nelore.

Em relação aos comprimentos de perna e de braço, dos efeitos genéticos aditivos raciais, apenas o componente individual foi significativo, e de sinal negativo. Com isso, novilhos Nelore apresentam carcaças com maiores comprimentos de ambas as características do que os Charolês. Enquanto que para os componentes do efeito genético heterótico, as estimativas foram positivas e de maior magnitude para o componente individual, ou seja, a heterozigose individual é proporcionalmente mais importante no incremento dos comprimentos de perna e de braço.

A estimativa dos efeitos genéticos pode ser interpretada ainda como informação estratégica, decisória, pois conforme Gregory e Cundiff (1980) e Koger (1980) pode servir de base para a determinação de quais raças e combinações entre estas devem ser utilizadas em um sistema de cruzamento.

Aspecto que justificaria a determinação das medidas métricas da carcaça seria por suas prováveis correlações com o peso de carcaça, sendo este último de valor econômico primordial para o produtor e indústria de matadouros-frigoríficos. Esta relação foi confirmada pelos coeficientes de correlação de Pearson significativos $(\mathrm{P}=0,0001)$ de 0,74 entre comprimento de carcaça e peso de carcaça fria (PCF), de 0,44 entre comprimento de perna e PCF e de 0,40 entre comprimento de braço e PCF. Todos os coeficientes de correlação foram positivos, indicando que maiores valores para medidas métricas estão relacionadas com carcaças frias mais pesadas, sendo com o comprimento de carcaça de maior magnitude. Este resultado justifica a preferência por indivíduos de biótipo denominado "moderno", priorizando o comprimento corporal.

Os resultados das médias ajustadas pelo Modelo I e os valores preditos pelo Modelo II para as características métricas da carcaça, de acordo com o grupo genético e sistema de acasalamento dos novilhos constam nas Tabelas 3 e 4 , respectivamente.

As estimativas dos efeitos genéticos do Modelo II apresentados na Tabela 2 apresentam a vantagem de possibilitar a determinação de valores preditos para grupos genéticos e sistemas de acasalamento não contemplados no estudo, como G5 e rotativo em equilíbrio. Exemplificando, o comprimento de carcaça de novilhos 2/3N1/3C (Rotativo em equilíbrio) foi predito da seguinte maneira: 122,4 $\mathrm{cm}=114,5 \mathrm{~cm}+[(7,15 \mathrm{~cm} * 0,333)+(1,44 \mathrm{~cm} *$ $0,667)+(4,47 \mathrm{~cm} * 0,667)+(2,32 \mathrm{~cm} * 0,667)]$. 
Tabela 3. Médias ajustadas pelo Modelo I, valores preditos pelo Modelo II e erros-padrão para características métricas da carcaça de acordo com o grupo genético dos novilhos.

\begin{tabular}{|c|c|c|c|c|c|c|}
\hline Modelo $^{1}$ & $\begin{array}{c}\text { Sistema de } \\
\text { acasalamento }\end{array}$ & Grupo genético & $\mathrm{n}$ & $\begin{array}{c}\text { Compr. de } \\
\text { carcaça, cm }\end{array}$ & $\begin{array}{l}\text { Compr. de } \\
\text { perna, cm }\end{array}$ & $\begin{array}{l}\text { Compr. de } \\
\text { braço, } \mathrm{cm}\end{array}$ \\
\hline \multirow[t]{15}{*}{ Modelo I } & \multirow[t]{3}{*}{ Definidos } & Charolês (C) & 184 & $123,3 a \pm 0,4$ & $67,4 b \pm 0,3$ & $38,3 b \pm 0,2$ \\
\hline & & Nelore (N) & 151 & $114,5 b+0,4$ & $71,4 a \pm 0,3$ & $40,1 \mathrm{a} \pm 0,2$ \\
\hline & & Valor t calculado & & 10,4 & 9,6 & 8,2 \\
\hline & \multirow[t]{3}{*}{ G1 } & $1 / 2 \mathrm{C} 1 / 2 \mathrm{~N}$ & 58 & $122,6 \mathrm{a} \pm 0,7$ & $72,1 \mathrm{a} \pm 0,5$ & $40,6 \mathrm{a} \pm 0,3$ \\
\hline & & $1 / 2 \mathrm{~N} 1 / 2 \mathrm{C}$ & 67 & $122,6 \mathrm{a} \pm 0,6$ & $71,1 \mathrm{a} \pm 0,5$ & $40,0 \mathrm{a} \pm 0,3$ \\
\hline & & Valor t calculado & & 3,0 & 1,5 & 1,5 \\
\hline & \multirow[t]{3}{*}{$\mathrm{G} 2$} & $3 / 4 \mathrm{C} 1 / 4 \mathrm{~N}$ & 137 & $125,0 \mathrm{a} \pm 0,4$ & $70,4 b \pm 0,3$ & $39,6 b \pm 0,2$ \\
\hline & & $3 / 4 \mathrm{~N} 1 / 4 \mathrm{C}$ & 107 & $121,5 b \pm 0,5$ & $72,5 \mathrm{a} \pm 0,4$ & $41,0 \mathrm{a} \pm 0,2$ \\
\hline & & Valor t calculado & & 3,0 & 4,2 & 5,1 \\
\hline & \multirow[t]{3}{*}{ G3 } & $5 / 8 \mathrm{C} 3 / 8 \mathrm{~N}$ & 49 & $124,1 \mathrm{a}+0,7$ & $71,6 b+0,6$ & $40,6 b+0,3$ \\
\hline & & $5 / 8 \mathrm{~N} 3 / 8 \mathrm{C}$ & 87 & $124,6 a \pm 0,6$ & $73,5 \mathrm{a} \pm 0,4$ & $42,1 \mathrm{a} \pm 0,2$ \\
\hline & & Valor t calculado & & 1,1 & 2,9 & 4,0 \\
\hline & \multirow[t]{3}{*}{ G4 } & $11 / 16 \mathrm{C} 5 / 16 \mathrm{~N}$ & 23 & $125,9 \mathrm{a}+1,1$ & $73,4 \mathrm{a} \pm 0,8$ & $40,1 \mathrm{a} \_0,5$ \\
\hline & & $11 / 16 \mathrm{~N} 5 / 16 \mathrm{C}$ & 13 & $123,5 \mathrm{a} \pm 1,4$ & $72,9 a \pm 1,1$ & $41,1 \mathrm{a} \pm 0,6$ \\
\hline & & Valor $\mathrm{t}$ calculado & & 1,4 & 0,3 & 1,3 \\
\hline \multirow[t]{14}{*}{ Modelo II } & \multirow[t]{2}{*}{ Definidos } & $\mathrm{C}$ & & 123,1 & 67,3 & 38,0 \\
\hline & & $\mathrm{N}$ & & 114,5 & 71,4 & 40,1 \\
\hline & \multirow[t]{2}{*}{ G1 } & $1 / 2 \mathrm{C} 1 / 2 \mathrm{~N}$ & & 122,5 & 72,3 & 40,5 \\
\hline & & $1 / 2 \mathrm{~N} 1 / 2 \mathrm{C}$ & & 124,0 & 72,0 & 40,8 \\
\hline & \multirow[t]{2}{*}{$\mathrm{G} 2$} & $3 / 4 \mathrm{C} 1 / 4 \mathrm{~N}$ & & 125,1 & 70,6 & 39,6 \\
\hline & & $3 / 4 \mathrm{~N} 1 / 4 \mathrm{C}$ & & 121,6 & 72,5 & 40,9 \\
\hline & \multirow[t]{2}{*}{ G3 } & $5 / 8 \mathrm{C} 3 / 8 \mathrm{~N}$ & & 123,8 & 71,4 & 40,0 \\
\hline & & $5 / 8 \mathrm{~N} 3 / 8 \mathrm{C}$ & & 122,8 & 72,3 & 40,8 \\
\hline & \multirow[t]{2}{*}{ G4 } & $11 / 16 \mathrm{C} 5 / 16 \mathrm{~N}$ & & 124,5 & 71,0 & 39,8 \\
\hline & & $11 / 16 \mathrm{~N} 5 / 16 \mathrm{C}$ & & 122,2 & 72,4 & 40,8 \\
\hline & \multirow[t]{2}{*}{ G5 } & $21 / 32 \mathrm{C} 11 / 32 \mathrm{~N}$ & & 124,2 & 71,2 & 39,9 \\
\hline & & $21 / 32 \mathrm{~N} 11 / 32 \mathrm{C}$ & & 122,5 & 72,3 & 40,8 \\
\hline & \multirow{2}{*}{$\begin{array}{l}\text { Rotativo em } \\
\text { equilíbrio }\end{array}$} & $2 / 3 \mathrm{C} 1 / 3 \mathrm{~N}$ & & 124,3 & 71,1 & 39,9 \\
\hline & & $2 / 3 \mathrm{~N} 1 / 3 \mathrm{C}$ & & 122,4 & 72,3 & 40,8 \\
\hline
\end{tabular}

a, b Médias para grupo genético dentro de sistema de acasalamento seguidas por letras diferentes na coluna, diferem $(\mathrm{P}<0,05)$ pelo teste $|\mathrm{t}|$.

${ }^{1}$ Modelo I: genotípico; Modelo II: genético aditivo-dominante.

Graus de liberdade do numerador $=5$. Graus de liberdade do erro $=848$.

Fonte: Elaboração dos autores.

Além disso, as estimativas dos efeitos genéticos são de grande valia na explicação das diferenças significativas entre os grupos genéticos dentro de sistema de acasalamento, bem como entre os sistemas de acasalamento. Pacheco et al. (2010) explicam que entre grupos genéticos dentro de sistema de acasalamento, a heterozigose é a mesma, logo, as diferenças significativas ocorrem devido às significâncias das estimativas dos efeitos genéticos aditivos raciais individual e materno combinados. Enquanto que entre os sistemas de acasalamento, a proporção racial Charolês x Nelore média é a mesma, portanto, as diferenças significativas ocorrem devido às significâncias das estimativas dos efeitos genéticos heteróticos individual e materno combinados. 
Em relação às médias para grupo genético dentro de sistema de acasalamento, verificam-se diferenças significativas $(\mathrm{P}<0,05)$ entre as raças definidas para todas as características avaliadas, confirmando os resultados das estimativas dos efeitos genéticos aditivos raciais apresentados na Tabela 2. Para os grupos genéticos cruzados, o comprimento de carcaça diferiu entre os da G2, enquanto que os comprimentos de perna e de braço foram distintos entre os da G2 e G3. Restle et al. (2000), Vaz et al. (2002) e Menezes et al. (2005) avaliaram características métricas da carcaça de novilhos oriundos do cruzamento rotativo Charolês x Nelore e verificaram resultados semelhantes aos do presente estudo, assim como Restle et al. (1999) verificaram incremento nos comprimentos de perna e de braço e redução no de carcaça de novilhos Hereford, Nelore e suas cruzas. Estes resultados indicam as diferenças morfológicas entre as raças utilizadas neste estudo, explicada por Berg e Butterfield (1976) por motivos de adaptabilidade.

Analisando sistemas de acasalamento da Tabela 4, verifica-se que as características métricas da carcaça foram inferiores para os definidos. Enquanto que pequenas variações foram verificadas entre os demais sistemas de acasalamento de mestiços. Nos estudos de Restle et al. (2000) e Menezes et al. (2005), que avaliaram as características da carcaça de novilhos terminados em confinamento e oriundos do cruzamento rotativo Charolês x Nelore, os resultados são concordantes com os do presente estudo, onde novilhos cruzados apresentam carcaças com maiores valores para características métricas em relação à média das definidas.

Tabela 4. Médias ajustadas pelo Modelo I, valores preditos pelo Modelo II e erros-padrão para características métricas da carcaça de acordo com o sistema de acasalamento dos novilhos.

\begin{tabular}{clccc}
\hline Modelo $^{1}$ & Sistema de acasalamento & Compr. de carcaça, cm & Compr. de perna, cm & Compr. de braço, cm \\
\hline Modelo I & Definidos & $118,9 \mathrm{c} \pm 0,3$ & $69,4 \mathrm{c} \pm 0,2$ & $39,2 \mathrm{c} \pm 0,1$ \\
& G1 & $122,6 \mathrm{~b} \pm 0,5$ & $71,6 \mathrm{ab} \pm 0,4$ & $40,3 \mathrm{~b} \pm 0,2$ \\
& G2 & $123,2 \mathrm{ab} \pm 0,3$ & $71,4 \mathrm{~b} \pm 0,3$ & $40,3 \mathrm{~b} \pm 0,1$ \\
& G3 & $124,3 \mathrm{a} \pm 0,5$ & $72,6 \mathrm{a} \pm 0,4$ & $41,3 \mathrm{a} \pm 0,2$ \\
& G4 & $124,7 \mathrm{a} \pm 0,9$ & $73,1 \mathrm{a} \pm 0,7$ & $40,6 \mathrm{ab} \pm 0,4$ \\
& Valor F calculado & 44,3 & 23,1 & 25,4 \\
& Definidos & 118,8 & 69,3 & 39,1 \\
& G1 & 123,3 & 72,1 & 40,6 \\
& G2 & 123,4 & 71,5 & 40,2 \\
& G3 & 123,3 & 71,8 & 40,4 \\
& G4 & 123,3 & 71,7 & 40,3 \\
& G5 & 123,3 & 71,8 & 40,4 \\
& Rotativo em equilíbrio & 123,3 & 71,7 & 40,4 \\
\hline
\end{tabular}

a, b, c Médias seguidas por letras diferentes na coluna, diferem $(\mathrm{P}<0,05)$ pelo teste $|\mathrm{t}|$.

${ }^{1}$ Modelo I: genotípico; Modelo II: genético aditivo-dominante.

Graus de liberdade do numerador $=4$. Graus de liberdade do erro $=848$.

Fonte: Elaboração dos autores.

Os valores de heterose, de acordo com o modelo (Tabela 5) foram expressos na unidade de medida da característica e em percentagem da média dos definidos. As heteroses foram positivas e significativas para todos os sistemas de acasalamento e para todas as características métricas avaliadas. Entre os modelos, os resultados foram muito próximos, embora com erros-padrão 
ligeiramente menores no Modelo II. Comparandose a diferença média entre os dois modelos os errospadrão, para as características avaliadas na Tabela 5, foram respectivamente: 38,$46 ; 28,9$ e $36 \% \mathrm{E}$ entre as características avaliadas, os valores de heterose em percentagem apresentaram pequena amplitude, oscilando entre 2,9 e 5,4\%. Avaliando características métricas da carcaça de novilhos oriundos do cruzamento rotativo Charolês x Nelore, Restle et al. (2000) relataram valores de heterose entre 3 e $6 \%$ (G1 e G2), enquanto que Menezes et al.
(2005) estimaram valores com amplitude um pouco superior, oscilando de 4 a 11,5\% (G2, G3 e G4). Os valores de heterose se expressam em animais mestiços entre zebuínos $\mathrm{x}$ taurinos, mostrando a importância da distância genética entre as raças que formam o cruzamento (PEROTTO; MOLETTA; CUBAS, 1999, 2000; PEROTTO; ABRAHÃO; KROETZ, 2001). Entre zebuínos, Ribeiro et al. (2008) citam que a heterose em animais mestiços Nelore e Guzerá é baixa, resultado da proximidade genética entre os dois genótipos.

Tabela 5. Heterose e erros-padrão (ep) para características métricas da carcaça de acordo com o modelo (Modelo I: genotípico; Modelo II: genético aditivo-dominante).

\begin{tabular}{|c|c|c|c|c|c|c|c|c|c|c|c|c|c|}
\hline \multirow{2}{*}{ Modelo } & \multirow{2}{*}{ Heterose } & \multicolumn{4}{|c|}{$\begin{array}{l}\text { Comprimento de } \\
\text { carcaça }\end{array}$} & \multicolumn{4}{|c|}{$\begin{array}{l}\text { Comprimento de } \\
\text { perna }\end{array}$} & \multicolumn{4}{|c|}{$\begin{array}{l}\text { Comprimento de } \\
\text { braço }\end{array}$} \\
\hline & & $\%$ & $\mathrm{~cm}$ & & $\begin{array}{l}\text { ep } \\
( \pm)\end{array}$ & $\%$ & $\mathrm{~cm}$ & & $\begin{array}{l}\text { ep } \\
( \pm)\end{array}$ & $\%$ & $\mathrm{~cm}$ & & $\begin{array}{l}\text { ep } \\
( \pm)\end{array}$ \\
\hline \multirow[t]{5}{*}{ Modelo I } & G1 & 3,1 & 3,7 & * & 0,6 & 3,2 & 2,2 & * & 0,4 & 2,8 & 1,1 & * & 0,2 \\
\hline & G2 & 3,6 & 4,4 & $*$ & 0,4 & 2,9 & 2,0 & * & 0,3 & 2,8 & 1,1 & * & 0,2 \\
\hline & G3 & 4,5 & 5,5 & $*$ & 0,6 & 4,6 & 3,2 & $*$ & 0,4 & 5,4 & 2,1 & $*$ & 0,2 \\
\hline & G4 & 4,9 & 5,9 & $*$ & 1,0 & 5,3 & 3,7 & $*$ & 0,7 & 3,6 & 1,4 & * & 0,4 \\
\hline & Retida $^{1}$ & 4,3 & 5,2 & $*$ & 0,5 & 4,3 & 3,0 & $*$ & 0,4 & 3,9 & 1,6 & $*$ & 0,2 \\
\hline \multicolumn{14}{|c|}{ Valor t calculado } \\
\hline & G1 & & 6,7 & & & & 5,1 & & & & 4,6 & & \\
\hline & G2 & & 10,2 & & & & 6,1 & & & & 6,1 & & \\
\hline & G3 & & 9,7 & & & & 7,3 & & & & 8,9 & & \\
\hline & G4 & & 6,1 & & & & 5,0 & & & & 3,5 & & \\
\hline & Retida & & 10,9 & & & & 8,1 & & & & 7,7 & & \\
\hline \multirow[t]{6}{*}{ Modelo II } & G1 & 3,8 & 4,5 & $*$ & 0,5 & 4,1 & 2,8 & $*$ & 0,4 & 4,0 & 1,6 & * & 0,2 \\
\hline & G2 & 3,8 & 4,6 & $*$ & 0,4 & 3,2 & 2,2 & * & 0,3 & 3,0 & 1,2 & * & 0,2 \\
\hline & G3 & 3,8 & 4,5 & $*$ & 0,4 & 3,6 & 2,5 & $*$ & 0,3 & 3,5 & 1,4 & $*$ & 0,1 \\
\hline & G4 & 3,8 & 4,5 & $*$ & 0,4 & 3,4 & 2,4 & $*$ & 0,3 & 3,3 & 1,3 & $*$ & 0,2 \\
\hline & G5 & 3,8 & 4,5 & $*$ & 0,3 & 3,5 & 2,4 & $*$ & 0,3 & 3,4 & 1,3 & $*$ & 0,1 \\
\hline & Retida $^{1}$ & 3,8 & 4,5 & $*$ & 0,4 & 3,5 & 2,4 & * & 0,3 & 3,4 & 1,3 & * & 0,1 \\
\hline \multicolumn{14}{|c|}{ Valor $t$ calculado } \\
\hline & G1 & & 9,2 & & & & 7,4 & & & & 7,6 & & \\
\hline & G2 & & 10,8 & & & & 6,8 & & & & 6,5 & & \\
\hline & G3 & & 12,9 & & & & 9,3 & & & & 9,2 & & \\
\hline & G4 & & 12,7 & & & & 8,5 & & & & 8,3 & & \\
\hline & G5 & & 13,1 & & & & 9,1 & & & & 9,0 & & \\
\hline & Retida & & 13,0 & & & & 9,0 & & & & 8,8 & & \\
\hline
\end{tabular}

\footnotetext{
${ }^{1}$ Aproximação do rotativo em equilíbrio.

$* \mathrm{P}<0,05$ pelo teste $|\mathrm{t}|$.

Graus de liberdade do erro $=848$.

Fonte: Elaboração dos autores.
} 
Analisando os contrastes cruzados vs Charolês e rotativo em equilíbrio vs Charolês (Tabela 6), verifica-se similaridade para comprimento de carcaça, e superioridade dos mestiços para comprimentos de perna e de braço. Trabalhando com as raças Charolês, Nelore e suas cruzas, Menezes et al. (2005) relataram diferenças superiores para os mestiços (G2, G3 e G4) em relação aos novilhos Charoleses para todas as características métricas. Enquanto que Restle et al. (1999), avaliando novilhos de diferentes genótipos de Hereford $\mathrm{x}$ Nelore, relataram superioridade dos mestiços em relação aos Hereford para comprimentos de perna e de braço.

Tabela 6. Contrastes e erros-padrão para características métricas da carcaça de acordo com o modelo (Modelo I: genotípico; Modelo II: genético aditivo-dominante).

\begin{tabular}{|c|c|c|c|c|c|c|c|}
\hline Modelo & Contrastes $^{1}$ & $\begin{array}{l}\text { Comprimento } \\
\text { de carcaça, } \mathrm{cm}\end{array}$ & $\begin{array}{c}\text { Valor t } \\
\text { calculado }\end{array}$ & $\begin{array}{l}\text { Comprimento } \\
\text { de perna, } \mathrm{cm}\end{array}$ & $\begin{array}{c}\text { Valor t } \\
\text { calculado }\end{array}$ & $\begin{array}{l}\text { Comprimento } \\
\text { de braço, } \mathrm{cm}\end{array}$ & $\begin{array}{c}\text { Valor t } \\
\text { calculado }\end{array}$ \\
\hline \multirow[t]{10}{*}{ Modelo I } & $\mathrm{G} 1 v_{s} \mathrm{C}$ & $-0,67 \pm 0,6$ & 1,1 & $4,23 \pm 0,5^{*}$ & 9,0 & $2,05 \pm 0,3 *$ & 7,9 \\
\hline & $\mathrm{G} 2$ vs $\mathrm{C}$ & $-0,04+0,5$ & 0,1 & $4,05 \pm 0,4^{*}$ & 10,8 & $2,05 \pm 0,2 *$ & 10,0 \\
\hline & $\mathrm{G} 3 v_{s} \mathrm{C}$ & $1,05 \pm 0,6^{* *}$ & 1,7 & $5,18 \pm 0,5^{*}$ & 11,0 & $3,08 \pm 0,3 *$ & 11,9 \\
\hline & $\mathrm{G} 4$ vs $\mathrm{C}$ & $1,44 \pm 1,0$ & 1,4 & $5,74 \pm 0,8^{*}$ & 7,5 & $2,37 \pm 0,4^{*}$ & 5,7 \\
\hline & $\begin{array}{l}\text { Cruzados } \\
\text { vs } \mathrm{C}\end{array}$ & $0,44 \pm 0,5$ & 0,9 & $4,81 \pm 0,4^{*}$ & 13,4 & $2,39 \pm 0,2 *$ & 12,1 \\
\hline & $\mathrm{G} 1 v_{s} \mathrm{~N}$ & $8,14 \pm 0,6^{*}$ & 13,0 & $0,20 \pm 0,5$ & 0,4 & $0,16 \pm 0,3$ & 0,6 \\
\hline & $\mathrm{G} 2 v s \mathrm{~N}$ & $8,77 \pm 0,5^{*}$ & 16,6 & $0,02 \pm 0,4$ & 0,1 & $0,17 \pm 0,2$ & 0,7 \\
\hline & $\mathrm{G} 3 v s \mathrm{~N}$ & $9,87 \pm 0,6^{*}$ & 15,4 & $1,16 \pm 0,5^{*}$ & 2,3 & $1,19 \pm 0,3 *$ & 4,3 \\
\hline & $\mathrm{G} 4 v s \mathrm{~N}$ & $10,26 \pm 1,0^{*}$ & 10,2 & $1,72 \pm 0,8^{*}$ & 2,2 & $0,49 \pm 0,4$ & 1,1 \\
\hline & $\begin{array}{l}\text { Cruzados } \\
\text { vs } \mathrm{N}\end{array}$ & $9,26 \pm 0,5^{*}$ & 18,6 & $0,78 \pm 0,4 *$ & 2,0 & $0,50 \pm 0,2 *$ & 2,3 \\
\hline \multirow[t]{12}{*}{ Modelo II } & $\mathrm{G} 1 v_{s} \mathrm{C}$ & $0,18 \pm 0,5$ & 0,3 & $4,88 \pm 0,4 *$ & 11,7 & $2,62 \pm 0,2 *$ & 11,3 \\
\hline & $\mathrm{G} 2$ vs $\mathrm{C}$ & $0,26 \pm 0,5$ & 0,5 & $4,30 \pm 0,4^{*}$ & 11,6 & $2,22 \pm 0,2 *$ & 10,8 \\
\hline & $\mathrm{G} 3$ vs $\mathrm{C}$ & $0,22 \pm 0,4$ & 0,5 & $4,59 \pm 0,3 *$ & 14,2 & $2,42+0,2 *$ & 13,5 \\
\hline & $\mathrm{G} 4 v s \mathrm{C}$ & $0,24 \pm 0,4$ & 0,5 & $4,44 \pm 0,3 *$ & 13,6 & $2,32 \pm 0,2 *$ & 12,7 \\
\hline & $\mathrm{G} 5$ vs $\mathrm{C}$ & $0,23 \pm 0,4$ & 0,5 & $4,51 \pm 0,3 *$ & 14,0 & $2,37 \pm 0,2 *$ & 13,3 \\
\hline & $\mathrm{RE}^{2}$ vs $\mathrm{C}$ & $0,23 \pm 0,4$ & 0,5 & $4,49 \pm 0,3 *$ & 13,9 & $2,35 \pm 0,2 *$ & 13,1 \\
\hline & $\mathrm{G} 1$ vs $\mathrm{N}$ & $8,77 \pm 0,6^{*}$ & 15,7 & $0,73 \pm 0,4^{*}$ & 1,7 & $0,54 \pm 0,2 *$ & 2,2 \\
\hline & $\mathrm{G} 2 v s \mathrm{~N}$ & $8,85 \pm 0,5^{*}$ & 17,4 & $0,15 \pm 0,4$ & 0,4 & $0,14 \pm 0,2$ & 0,6 \\
\hline & $\mathrm{G} 3 v s \mathrm{~N}$ & $8,81 \pm 0,5^{*}$ & 19,6 & $0,44 \pm 0,4$ & 1,2 & $0,34 \pm 0,2 * *$ & 1,7 \\
\hline & $\mathrm{G} 4 v s \mathrm{~N}$ & $8,83 \pm 0,5 *$ & 19,3 & $0,29 \pm 0,4$ & 0,8 & $0,24 \pm 0,2$ & 1,2 \\
\hline & $\mathrm{G} 5 v s \mathrm{~N}$ & $8,82 \pm 0,5^{*}$ & 19,7 & $0,36 \pm 0,4$ & 1,0 & $0,29 \pm 0,2$ & 1,5 \\
\hline & $\mathrm{RE}^{2} v s \mathrm{~N}$ & $8,83 \pm 0,5^{*}$ & 19,7 & $0,34 \pm 0,4$ & 0,9 & $0,28 \pm 0,2$ & 1,4 \\
\hline
\end{tabular}

${ }^{1}$ Cruzados $=(\mathrm{G} 1+\mathrm{G} 2+\mathrm{G} 3+\mathrm{G} 4) / 4$.

${ }^{2}$ Rotativo em equilíbrio.

* $\mathrm{P}<0,05 ; * * \mathrm{P}<0,10$ pelo teste $|\mathrm{t}|$.

Graus de liberdade do erro Modelo I $=848$. Graus de liberdade do erro Modelo II $=854$.

Fonte: Elaboração dos autores.

Para os contrastes cruzados vs $\mathrm{N}$ e rotativo em equilíbrio vs $\mathrm{N}$, houve superioridade dos mestiços para comprimento de carcaça e resultado controverso para as demais características métricas.
No estudo de Menezes et al. (2005), o contraste mestiços (G2, G3 e G4) vs Nelore foi positivo para todas as características métricas da carcaça. 
A contribuição dos efeitos de epistasia e ligação gênica na variação das características métricas da carcaça avaliadas foram significativas $(\mathrm{P}<0,05)$, no entanto, com valores de percentagem de redução pouco expressivos (4\%; Tabela 7). Avaliando o cruzamento rotativo Angus, Polled Hereford e Santa Gertrudis, Neville Junior, Mullinix Junior e McCormick (1984) estimaram 2\% $\quad(\mathrm{P}<0,05)$ de redução para comprimento de carcaça, e concluíram que efeitos de epistasia e ligação gênica aparentam ter maior influência em características de desempenho pós-desmame e de carcaça do que em características reprodutivas. No entanto, demais estudos avaliando o cruzamento rotativo Charolês $\mathrm{x}$ Nelore relataram influência irrelevante dos efeitos genéticos não aditivos que não os de dominância em características que expressam musculosidade da carcaça (Pacheco et al., 2010).

Tabela 7. Graus de liberdade do erro (GLE), soma de quadrados do erro (SQE), percentagem de redução, coeficiente de determinação $\left(\mathrm{R}^{2}\right)$ e coeficiente de correlação de Pearson (r) entre modelos ${ }^{1}$ (I e II) de acordo com as características avaliadas.

\begin{tabular}{|c|c|c|c|c|c|c|c|c|c|c|c|}
\hline \multirow[b]{2}{*}{$\begin{array}{l}\text { Caracte- } \\
\text { rísticas }\end{array}$} & \multicolumn{3}{|c|}{ GLE } & \multicolumn{3}{|c|}{ SQE } & \multicolumn{2}{|c|}{ Redução $^{2}$} & \multicolumn{2}{|c|}{$\mathrm{R}^{2}$} & \multirow{2}{*}{$\begin{array}{c}\mathrm{r} \text { entre } \\
\text { mode- } \\
\text { los }\end{array}$} \\
\hline & II & I & $\begin{array}{l}\text { Redu- } \\
\text { ção }\end{array}$ & II & I & $\begin{array}{l}\text { Redu- } \\
\text { ção }\end{array}$ & $\%$ & $\operatorname{Pr}>F$ & II & I & \\
\hline C. carcaça & 854 & 849 & 5 & 19.801 & 19.466 & 335 & 1,72 & 0,013 & 0,51 & 0,52 & $0,96^{*}$ \\
\hline C. perna & 854 & 849 & 5 & 11.826 & 11.588 & 238 & 2,05 & 0,004 & 0,37 & 0,38 & $0,93^{*}$ \\
\hline C. braço & 853 & 848 & 5 & 3.639 & 3.512 & 128 & 3,64 & 0,001 & 0,39 & 0,41 & $0,93 *$ \\
\hline
\end{tabular}

${ }^{1}$ Modelo I: genotípico; Modelo II: genético aditivo-dominante.

${ }^{2}$ Redução (em percentagem) representa a contribuição dos efeitos de epistasia e ligação gênica na variação da característica.

* $\mathrm{P}<0,05$ pelo teste $|\mathrm{r}|$.

Fonte: Elaboração dos autores.

Apesar do Modelo I englobar os efeitos aditivos devido à raça e todos os não aditivos, pode-se afirmar que os dois modelos apresentam eficiência similar, conforme demonstrado pela proximidade dos valores dos $\mathrm{R}^{2}$ de ambos os modelos para cada característica. Além disso, os coeficientes de correlação de Pearson entre os valores obtidos pelo Modelo I e pelo Modelo II foram de elevada magnitude para todas as características. No entanto, considerando que a heterozigose materna foi significativamente importante para as características métricas da carcaça avaliadas, sugere-se que o Modelo II deve ser preferencialmente adotado em relação ao Modelo I, pois tem a possibilidade de estimar este efeito genético, diferentemente do Modelo I, cuja estimativa foi limitada pelo tipo de modelo adotado (Tabela 2).

\section{Conclusões}

Das características métricas da carcaça avaliadas, o comprimento de carcaça é o mais influenciado pelos efeitos genéticos aditivos raciais e heteróticos, sendo o componente individual de maior importância do que o materno.

A raça Charolesa apresenta maior comprimento de carcaça e menores de perna e de braço do que a raça Nelore.

A heterose é positiva e, quando expressa em percentagem, os valores são muito próximos para todas as características métricas.

O sistema de cruzamento rotativo entre as raças Charolesa e Nelore é beneficiado pelos efeitos de heterose e complementaridade racial nas características métricas da carcaça de novilhos submetidos a sistema de produção visando o abate aos dois anos de idade. 


\section{Referências}

ARTHUR, P. F.; HEARSHAW, H.; STEPHENSON, P. D. Direct and maternal additive and heterosis effect from crossing Bos indicus and Bos taurus cattle: Cow and calf performance in two environments. Livestock Production Science, Amsterdam, v. 57, n. 3, p. 231-241, 1999.

BERG, R. T.; BUTTERFIELD, R. M. New concepts of cattle growth. Sydney: Sydney University Press, 1976. $240 \mathrm{p}$.

CLÍMACO, S. M.; RIBEIRO, E. L. A.; MIZUBUTI, I. Y.; SILVA, L. D. F. da; BARBOSA, M. A. A. F.; BRIDI, A. M. Desempenho e características de carcaça de bovinos de corte de quatro grupos genéticos terminados em confinamento. Revista Brasileira de Zootecnia, Viçosa, MG, v. 40, n. 7, p. 1562-1567, 2011.

DeROUEN, S. M.; FRANKE, D. E.; BIDNER, T. D.; BLOUIN, D. C. Two-, three-, and four-breed rotational crossbreeding of beef cattle: carcass traits. Journal of Animal Science, Champaign, v. 70, n. 12, p. 3665-3676, 1992.

DILLARD, E. U.; RODRIGUEZ, O.; ROBINSON, O. W. Estimation of additive and nonadditive direct and maternal effects from crossbreeding beef cattle. Journal of Animal Science, Champaign, v. 50, n. 4, p. 653-663, 1980.

GREGORY, K. E.; CUNDIFF, L. V. Crossbreeding in beef cattle: evaluation of systems. Journal of Animal Science, Champaign, v. 51, n. 5, p. 1224-1243, 1980.

KOGER, M. Effective crossbreeding systems utilizing zebu cattle. Journal of Animal Science, Champaign, v. 50, n. 6, p. 1213-1220, 1980.

MENEZES, L. F. G.; RESTLE, J.; BRONDANI, I. L.; ALVES FILHO, D. C.; KUSS, F.; SILVEIRA, M. F. da; AMARAL, G. A. do. Características da carcaça de novilhos de gerações avançadas do cruzamento alternado entre as raças Charolês e Nelore, terminados em confinamento. Revista Brasileira de Zootecnia, Viçosa, MG, v. 34, n. 3, p. 934-945, 2005.

NEVILLE JUNIOR, W. E.; MULLINIX JUNIOR, B. G.; McCORMICK, W. C. Grading and rotational crossbreeding of beef cattle. III. Postweaning and carcass traits of steers. Journal of Animal Science, Champaign, v. 58, n. 1, p. 47-56, 1984.

PACHECO, P. S.; RESTLE, J.; BRONDANI, I. L.; ALVES FILHO, D. C.; PADUA, J. T.; MIOTTO, F. R. C. Grupo genético, sistema de acasalamento e efeitos genéticos aditivos e não-aditivos nas características que expressam musculosidade da carcaça de novilhos oriundos do cruzamento rotativo Charolês x Nelore. Revista Brasileira de Zootecnia, Viçosa, MG, v. 39, n. 3, p. 494-502, 2010.
PEROTTO, D.; ABRAHÃO, J. J. S.; KROETZ, I. A. Produtividade à desmama de novilhas nelore e fl Bos taurus x nelore e Bos indicus x nelore. Revista Brasileira de Zootecnia, Viçosa, MG, v. 30, n. 6, p. 1712-1719, 2001.

PEROTTO, D.; MOLETTA, J. L.; CUBAS, A. C. Características da carcaça de bovinos Canchim e Aberdeen Angus e de seus cruzamentos recíprocos terminados em confinamento. Ciência Rural, Santa Maria, v. 29, n. 2, p. 331-338, 1999.

Características quantitativas da carcaça de bovinos charolês, caracu e cruzamentos recíprocos terminados em confinamento. Revista Brasileira de Zootecnia, Viçosa, MG, v. 29, n. 1, p. 117-124, 2000.

PEROTTO, D.; MOLETTA, J. L.; LESSKIU, C. Desempenho em confinamento de machos bovinos inteiros Canchim, Aberdeen Angus e cruzamentos recíprocos. Ciência Rural, Santa Maria, v. 32, n. 4, p. 669-674, 2002.

RESTLE, J.; VAZ, F. N.; FEIJÓ, G. L. D.; BRONDANI, I. L.; ALVES FILHO, D. C.; BERNARDES, R. A. L. C.; FATURI, C.; PACHECO, P. S. Características de carcaça de bovinos de corte inteiros ou castrados de diferentes composições raciais Charolês x Nelore. Revista Brasileira de Zootecnia, Viçosa, MG, v. 29, n. 5, p. 1371-1379, 2000.

RESTLE, J.; VAZ, F. N.; QUADROS, A. R. B.; MÜLLER, L. Características de carcaça e da carne de novilhos de diferentes genótipos de Hereford $\mathrm{x}$ Nelore. Revista Brasileira de Zootecnia, Viçosa, MG, v. 28, n. 6, p. 1245-1251, 1999.

RIBEIRO, E. L. A.; HERNANDEZ, J. A.; ZANELLA, E. L.; MIZUBUTI, I. Y.; SILVA, L. D. F. da; REEVES, J. J. Desempenho e características de carcaça de bovinos de diferentes grupos genéticos. Revista Brasileira de Zootecnia, Viçosa, MG, v. 37, n. 9, p. 1669-1673, 2008.

SHAPIRO, S. S.; WILK, M. B. An analysis of variance test for normality (complete samples). Biometrika, London, v. 52, n. 3-4, p. 591-611, 1965.

STATISTICAL ANALYSIS SYSTEMS - SAS. Sas Institute. User's guide. Version 9.4. North Caroline: SAS Institute Corporation, 2013.

VAZ, F. N.; RESTLE, J.; ALVES FILHO, D. C.; BRONDANI, I. L.; PASCOAL, L. L.; VAZ, R. Z.; PEIXOTO, L. A. O. Características de carcaça e da carne de novilhos filhos de vacas $1 / 2$ Nelore $1 / 2$ Charolês e $1 / 2$ Charolês $1 / 2$ Nelore acasaladas com touros Charolês ou Nelore. Revista Brasileira de Zootecnia, Viçosa, MG, v. 31, n. 4, p. 1734-1743, 2002. 